\title{
Experimental particle paths and drift velocity in steep waves at finite water depth
}

\author{
John Grue ${ }^{1 \dagger}$ and Jostein Kolaas ${ }^{1}$ \\ ${ }^{1}$ Mechanics Division, Department of Mathematics, University of Oslo, Oslo, Norway
}

(Received xx; revised xx; accepted xx)

The Lagrangian paths, horizontal Lagrangian drift velocity, $U_{L}$, and the Lagrangian excess period, $T_{L}-T_{0}$, where $T_{L}$ is the Lagrangian period and $T_{0}$ the Eulerian linear period, are obtained by particle tracking velocimetry (PTV) in non-breaking periodic laboratory waves at a finite water depth of $h=0.2 \mathrm{~m}$, wave height of $H=0.49 h$ and wavenumber of $k=0.785 / h$. Both $U_{L}$ and $T_{L}-T_{0}$ are functions of the average vertical position of the paths, $\bar{Y}$, where $-1<\bar{Y} / h<0$. The functional relationships $U_{L}(\bar{Y})$ and $T_{L}-T_{0}=f(\bar{Y})$ are very similar. Comparisons to calculations by the inviscid strongly nonlinear Fenton method and the second-order theory show that the streaming velocities in the boundary layers below the wave surface and above the fluid bottom contribute to a strongly enhanced forward drift velocity and excess period. The experimental drift velocity shear becomes more than twice that obtained by the Fenton method which again is approximately twice that of the second-order theory close to the surface. There is no mass flux of the periodic experimental waves and no pressure gradient. The results from a total number of 80000 experimental particle paths in the different phases and vertical positions of the waves show a strong collapse. The particle paths are closed at the two vertical positions where $U_{L}=0$.

\section{Introduction}

The mean wave drift - the Stokes drift - was introduced by Stokes (1847). The mathematical theory was in water of finite depth generalized to include the secondary streaming effect caused by the boundary layers at the bottom and surface by LonguetHiggins (1953). The theory explained a set of measurements by Bagnold (1947) where the drift at the bottom boundary layer along the wave propagation direction was strong enough to cause a backward drift at all of the other vertical positions of the water column. The Stokes drift contributes to the momentum and energy fluxes at the ocean surface. E.g. Ardhuin et al. (2009) have used HF radar recordings in the field to decompose the wave drift into a quasi-Eulerian current and a Stokes drift estimating their respective contributions to the Ekman layer. In wave and ocean current interaction models, the contribution by the Stokes drift is expressed in terms of a vortex force, material advection terms and a gradient term in the turbulent kinetic energy equation, where the wave effects in turn generate the Langmuir circulation in the oceanic boundary layer, see Sullivan and McWilliams (2010). A generalized Lagrangian mean flow theory for the interaction between a wave motion and a mean flow was developed by Andrews and McIntyre (1978). A modification of the theory by Groeneweg and Klopman (1998) was tested for the case of surface waves interacting with non-turbulent and turbulent shear flows comparing to experiments. Return flows will develop under a finite packet of waves due to radiation stress gradients, see Longuet-Higgins and Stewart (1962) and McIntyre (1981).

$\dagger$ Email address for correspondence: johng@math.uio.no 
Monismith et al. (2007) have presented several sets of laboratory measurements where the averaged Lagrangian wave drift velocity has been found to be zero across all of the water column. This implies that an Eulerian mean velocity locally cancels the Stokes drift in those experiments. The results of Monismith et al. have primarily been found in the combined cases with waves propagating on constant and sheared currents, where in the experiments by Klopman, referred to in Groeneweg and Klopman (1998), and those of Nepf et al. (1995), the underlying channel flows were turbulent, where the effects of the bottom boundary layer could be felt throughout the depth at eariler distance and time compared to the case where waves propagate along a fluid layer otherwise at rest. However, not all experiments reported in the literature conform with the measurements obtaining a vanishing Lagrangian wave drift velocity, where examples include the experiments referenced in Groeneweg and Klopman (1998), see also the references in Monismith et al. (2007). The differences in the results point to conflicts between the various experimental observations including the differences in the nonlinear wave and mean flow interaction processes as well as the boundary conditions.

At finite water depth, with $k h \rightarrow 1$ or less (where $k$ is the wavenumber and $h$ the water depth), Monismith et al. (2007) suggest that an interplay between the wave motion and the bottom boundary layer may alter the vertical variation of the Lagrangian velocity. In which way the changes occur await experimental clarification, however. It is rather uncertain if the mean particle velocity would cancel exactly at all vertical positions as in the deeper water cases (larger $k h$ ) reported by Monismith et al. (2007). Theoretical calculations of the particle motion in nonlinear water waves at finite depth by, e.g., Constantin (2006), Hsu, Chen and Wang (2010) and Constantin (2015), do not address the contributions by the boundary layers at the bottom and wave surface.

We here provide wave tank measurements of the Lagrangian drift velocity in a nonbreaking strongly nonlinear periodic wave train at finite water depth with $k h=0.785$. The experiment has been previously published in Grue, Kolaas and Jensen (2014) where the focus was directed to the maximum possible orbital velocity in the steep experimental waves. The experimental data are here reprocessed obtaining the Lagrangian particle paths, drift velocity and its shear. Particularly the drift caused by the streaming in the boundary layers at the bottom and below the surface are investigated. By means of particle tracking velocimetry (PTV) we record the wave field at an early position of the wave tank when the wave train has become periodic and before the waves interact with the beach at the end opposite to the wave maker. The waves we measure propagate in practice in an open-ended channel where no external pressure gradient exists.

Section 2 describes the experiments, methods and the particle paths, section 3 the Lagrangian period, drift velocity and its shear, while section 4 is a conclusion.

\section{Experiments and methods}

The waves are generated in a $25 \mathrm{~m}$ long and $0.5 \mathrm{~m}$ wide wave tank in the Hydrodynamics Laboratory at the University of Oslo. The water depth is $h=0.2 \mathrm{~m}$. Recordings of the waves generated at a paddle frequency of $1 / T_{0}=0.8 \mathrm{~Hz}\left(T_{0}\right.$ the period) are obtained at a distance from the wave maker of $4.4 \mathrm{~m}=22 \mathrm{~h}$. The elevation and velocity field become periodic, after a short transient build-up, where the recordings are made before the leading part of the wave train has interferred with the beach at the other end of the tank. The fluid velocities are obtained by PTV using the code DigiFlow, see Dalziel (1992). The slightly tilted camera has a field of view (FOV) extending $0.206 \mathrm{~m}$ vertically by $0.21 \mathrm{~m}$ along the upper horizontal and $0.198 \mathrm{~m}$ along the lower horizontal, with a resolution of 1024 by 1024 pixels, and a sampling rate of 1500 frames per second (fps). 
The recordings are obtained in four time intervals, each lasting for 3.9 seconds. With the camera at two vertical positions, totally eight sequences of the PTV are recorded as indicated by the white lines in figure 1a.

The trough to crest heigth $H$ of the eight crests from 8 to $17.7 \mathrm{~s}$ has a maximum of $H=$ $0.49 h$, average of $\bar{H}=0.47 h$ and a standard deviation of $3 \%$. The maximum horizontal orbital velocity is $0.50 \sqrt{g h}$ (where $g$ denotes the acceleration of gravity). We compare the non-breaking experimental waves to the strongly nonlinear Fenton (1988) method where in the latter 20 terms of the expansions are used and the vertically averaged Eulerian volume flux is put to zero. The similar theoretical wave with $H / h=0.49$ gives a calculated maximum of the horizontal orbital velocity of $0.42 \sqrt{g h}$. This velocity is approximately $16 \%$ smaller than the experimental value. The discrepancy between the experimental and theoretical velocity illustrates the contribution by the small parasittic second-harmonic free waves riding on the mother wave train, where the parasittic waves originate from the finite excursions of the wave maker. We note that the parasittic free wave is moving at the double frequency $2 \omega_{0}$ and the wavenumber $k_{2}$ which are connected by the linear dispersion relation, i.e., $\left(2 \omega_{0}\right)^{2}=g k_{2} \tanh \left(k_{2} h\right)$, where $\omega_{0}=2 \pi / T_{0}$. Since the frequency is twice that of the mother wave, the wave height of the parasittic waves is approximately $16 \%$ divided by two giving $8 \%$ compared to the mother wave. The parasittic wave amplitude may alteratively be estimated from the difference of the wave height of the unpolluted wave crest number three in figure $1 \mathrm{a}$, of $H / h \sim 0.435$, which arrives before the slower propagating parasittic wave train, and the subsequent wave crests number 4 to 11 . This estimate confirms a parasittic wave amplitude of approximately $8 \%$ of that of the mother wave. We note that the contribution by the parasittic wave train to the drift velocity does not couple to the mother wave and its higher harmonic bound modes where the relative contribution is proportional to the relative amplitude squared, i.e. $0.08^{2} \simeq 0.006$.

\subsection{Particle paths}

Particle paths are calculated in time window number 1 for $t \sim 9.9-13.8$ seconds and time window number 2 for $t \sim 13.8-17.7$ seconds, see figure 1a. In each single image a number of approximately $10^{4}$ particles are identified by the PTV. With a rate of $1500 \mathrm{fps}$ a total number of 120 million individual particles is identified in each time window including recordings in the upper and lower water column. Particle path fragments through time intervals exceeding a lower threshold of $33 d t=0.02$ seconds, totally 0.21 (and 0.23 ) million such fragments form the basis of the path calculations in the time window 1 (and 2). A small number of 34 (and 12) particles through the entire recording in time window 1 (and 2) are traced. The particle path through an entire wave period emerge by an interpolation combining several fragments. Smoothing of the particle paths are obtained using a 31 points second order Savitzky-Golay Filter in time (Savitzky and Golay, 1964). For the interpolation of the paths a third order local polynomial approximation in space is used where the 250 nearest points are weighted by the distance from the evaluation point (Ruppert and Wand, 1994). Particle paths, obtained for various wave phases by integrating one period both backward and forward in time, see figure $2 \mathrm{a}$, show a net forward motion right below the wave surface, a net backward drift in the middle of the wave column and then a net forward drift close to the bottom. Different from theoretical calculations, e.g., Constantin (2006), closed particle paths occur at the two vertical positions where the Lagrangian drift velocity $\left(U_{L}\right)$ is zero, where $U_{L}$ is defined below. The use of interpolation polynomials implies that Gibbs phenomenon appears at the boundaries of the images, near the surface, bottom and where the velocity gradients are strong. 


\section{Lagrangian period and drift velocity}

For convenience, horizontal axis $x$ and vertical axis $y$ with $y=0$ at the water level at rest are introduced. In order to accurately evaluate the Lagrangian drift, the path of the backward time integration is translated in the $x$ - and $y$-direction until it fits with the path of the forward time integration, evaluating the distance function

$$
d=\frac{1}{T_{L}} \int_{t_{0}-T_{L} / 2}^{t_{0}+T_{L} / 2} d t \sqrt{\left(x_{L}\left(t+T_{L}\right)-x_{L}(t)-x_{0}\right)^{2}+\left(y_{L}\left(t+T_{L}\right)-y_{L}(t)-y_{0}\right)^{2}} .
$$

Here, $x_{L}$ and $y_{L}$ denote the Lagrangian horizontal and vertical positions, respectively. Values of the Lagrangian period $T_{L}$ and the drift distances $x_{0}$ and $y_{0}$ are obtained by minimizing the function $d$ in (3.1), see figure $2 \mathrm{~b}$. The starting time $t_{0}$ (the phase) of the integration is varied within the time windows one and two. The resulting particle paths are compressed in the lower part and uplifted in the central upper part, due to the strong nonlinearity. The paths are also somewhat tilted and is an effect of the shorter parasittic second harmonic waves riding on the mother wave. They contribute to a weak shear deformation of the path, an effect that may be calculated by a superposition of the velocity field due to the parasittic waves. The tilt varies according to the phase (or initial time $t_{0}$ ). A small observed vertical drift velocity, both positive and negative, is one order of magnitude smaller than the horizontal drift velocity and indicates the accuracy of the experiments, see figure $4 \mathrm{c}, \mathrm{d}$, and is further commented on below.

It is convenient to define the average vertical coordinate of a loop by

$$
\bar{Y}=\frac{1}{T_{L}} \int_{t_{0}-T_{L} / 2}^{t_{0}+T_{L} / 2} y_{L}(t) d t
$$

A number of 75 different initial times $t_{0}$ (phases) at approximately $0.5 \cdot 10^{3}$ points along the vertical, in totally $\sim 4 \cdot 10^{4}$ combinations in each time window, are used in the evaluations of the functions $\bar{Y}, T_{L}, x_{0}$ and $y_{0}$. It is noted that the vertical coordinate $\bar{Y}$ spans the range $-1^{+}<\bar{Y} / h \leqslant-\epsilon$ where $-\epsilon \simeq 0^{-}$. The components of the drift velocity are obtained by $U_{L}=x_{0} / T_{L}$ and $V_{L}=y_{0} / T_{L}$.

The experimental Lagrangian excess period, $T_{L}-T_{0}$, and the horizontal drift velocity as functions of the vertical position $\bar{Y}$ are illustrated in figure 3 for the two time windows. Both variables exhibit a strong collapse of the experimental data, particularly $U_{L}$. The mean Eulerian velocity, $U_{E}(y)$, is obtained in the figure as well. The experimental volume flux is obtained by evaluating the integral

$$
\bar{U}_{L}=\frac{1}{h} \int_{-h}^{0} U_{L}(\bar{Y}) d \bar{Y}
$$

where extrapolation of $U_{L}$ near $\bar{Y}=0$ and $\bar{Y}=-h$ is used. The integration gives $\bar{U}_{L} / \sqrt{g h} \simeq-0.2 \cdot 10^{-3}$ for time window 1 and $\bar{U}_{L} / \sqrt{g h} \simeq-1.3 \cdot 10^{-3}$ for time window 2 and means that the mass flux in practice is zero in the experimental waves. The excess period and horizontal drift velocity obtained in the experiments differ significantly from the inviscid Fenton method and the second-order theory. Note that the Fenton method gives a vertically averaged Lagrangian drift velocity of $\bar{U}_{L, F e n t o n} \sim-0.9 \cdot 10^{-3} \sqrt{g h}$. The second-order theory obtains the drift velocity by, see Longuet-Higgins (1953),

$$
\bar{U}_{2 n d}=\frac{a^{2} \omega k \cosh 2 k(y+h)}{2 \sinh ^{2} k h}-\frac{a^{2} \omega}{2 h} \operatorname{coth}(k h),
$$

in which the vertical average is assumed to be zero and the amplitude is taken as $a=H / 2$. Based on the second-order theory, Longuet-Higgins (1953) obtained mathematically 
[]

\begin{tabular}{|c|c|c|c|c|}
\hline & \multicolumn{2}{|c|}{ Experiments } & \multicolumn{2}{|c|}{ Inviscid theory } \\
\hline & | t.w.1 & |t.w.2 | av. | & Fenton & nd-order \\
\hline$U_{L} / \sqrt{g h}$ & | 0.048 & $|0.038| 0.043 \mid$ & 0.031 & 0.023 \\
\hline $\mid \partial U_{L} / \partial \bar{Y} \sqrt{h / g}$ & 0.41 & $0.29|0.35|$ & 0.15 & 0.08 \\
\hline
\end{tabular}

TABLE 1. The drift velocity and its gradient at $\bar{Y}=0$. Experiments in time windows 1 (t.w.1), 2 (t.w.2) and the average of the two (av.). Same obtained by the Fenton theory and second-order theory.

the streaming effect due to the oscillatory boundary layer at the bottom giving a mass-transport velocity of $\Delta U_{L}=(5 / 4) a^{2} \omega k / \sinh ^{2}(k h)(\simeq 0.056 \sqrt{g h}$ with the present parameters). The similar analysis of the streaming effect below the surface gives a drift velocity gradient that is twice that obtained from (3.4), where both results are independent of the viscosity. The doubling of the drift velocity gradient below the surface, derived in the context of the second-order theory, was experimentally confirmed by Longuet-Higgins (1960). An even larger excess of the drift velocity gradient at $\bar{Y}=0$ is measured here in the strongly nonlinear waves. Comparing the experimental and inviscid drift velocities right below the wave surface at $\bar{Y}=0$ the experimental $U_{L}$ is $50 \%$ higher than the prediction by the Fenton method, and $100 \%$ higher than the second-order theory, approximately, see table 1 . Above the bottom the increase in the experimental drift velocity due to the streaming effect is $\Delta U_{L} / \sqrt{g h} \simeq 0.020$ in time window 1 and 0.023 in time window 2, both about the half of the prediction by Longuet-Higgins (1953). Note that the dimensionless functions $T_{L} / T_{0}-1=f(\bar{Y})$ and $U_{L}(\bar{Y}) / \sqrt{g h}$ are rather similar through the entire water column, where both the drift velocity and the Lagrangian period are significantly modified by the streaming effects at $\bar{Y}=0$ and $\bar{Y}=-h$ as well as the nonlinearity of the waves.

The Eulerian mean horizontal velocity $\left(U_{E}\right)$ obtained by a gliding time average exhibit a steady state for $t>9$ seconds at positions close to the bottom $(y / h=-0.994,-0.96)$ (note a small increase at -0.996 ) and below the surface at $y / h=-0.15$ (figure $1 \mathrm{~b}$ ) (For $y$ above the troughs $U_{E}$ is not meaningful.) While $y / h=-0.994$ corresponds to a level of $1.2 \mathrm{~mm}$ above the bottom, the estimated boundary layer thickness is $\delta=\sqrt{2 \omega / \nu} \simeq 3$ $\mathrm{mm}$. However, the effects of the streaming above the bottom and below the surface extend further into the body of the water layer, above the bottom up to $y / h \simeq-0.92$, and below the surface down to $y / h \simeq-0.2$, see below. We note that Longuet-Higgins' solution does not apply to the present experiments since the wave amplitude (of $H / 2=49 \mathrm{~mm}$ ) is much bigger than the boundary layer thickness of $3 \mathrm{~mm}$. The potential effect of the surface tension to the surface boundary layer was discussed by Weber and Saetra (1995). Particularly their figure 3 shows that the velocity is reduced right below the surface (corresponding to $\bar{Y} / h>-0.12$ ) for the conditions in the present experiments where an estimate of their parameter $\alpha$ in their eq. (27) is close to 300, contributing to an enhanced shear for $\bar{Y} / h<-0.12$ and a reduced shear for $-0.12<\bar{Y} / h<0$.

By evaluating the vertical gradient of $U_{L}$ it is evident that the inviscid Fenton method and the second order theory represent the same physics as observed in the experiments, 
in the middle of the water column, for $-0.9<\bar{Y} / h<-0.2$, see figure 4a,b. Near the surface $(-0.2<\bar{Y} / h<0)$ additional contributions are due to the combined effects of the streaming and nonlinearity. At the bottom $(-1<\bar{Y} / h<-0.9)$ the notable differences are caused by the boundary layer induced streaming. The experimental dimensionless gradient at $\bar{Y}=0$ is 0.40 in time window 1 and 0.28 in time window 2, with an average of 0.34 , while the Fenton method gives a value that is less than half of the experimental, and the second order theory about a quarter of the experimental, see table 1 . At the bottom the nondimensional shear is down to -0.98 and -1.15 in the two time windows (figure $4 \mathrm{a}, \mathrm{b}$ ). Evaluation of the vertical drift velocity provides an accuracy test of the experiments, since this velocity should be zero. The results show that $V_{L}$ is one order of magnitude smaller than $U_{L}$ (figure 4c,d). Right below the wave surface a small positive $V_{L}$ is up to $8 \%$ of $U_{L}$, while for $\bar{Y} / h<-0.2$ the velocity is small and negative, with a magnitude of $5-10 \%$ of $U_{L}$. We note that a small unsteadiness of the wave train in the form of an increased amplitude contributes to a small positive $V_{L}$ close to the surface. Potential contributions by the return flow in the water column to a negative/positive $V_{L}$ await further exploration.

We note note that extensions of the Gerstner's wave theory to a finite water depth, where by definition the particle paths are closed and $U_{L}=0$ - contrary to the present measurements, and where further the vorticity is non-zero, have been developed as model for irregular waves, with favorable comparison of the theoretical horizontal velocity profile below the wave crest and LDV-experiments, see Gjøsund (2003).

The average elevation within the experimental wave train is unaltered by the waves.

\section{Conclusions}

In experimental non-breaking periodic waves at finite water depth of $k h=0.785$ and $H / h=0.49$, we obtain by PTV, from the Lagrangian paths, the horizontal drift velocity, $U_{L}$, and the Lagrangian excess period, $T_{L}-T_{0}$, where $U_{L}$ and $T_{L}-T_{0}$ are functions of the average vertical position of the paths, $\bar{Y}$. The experimental results in the different phases and vertical positions show a collapse of the data. The drift velocity and excess period have similar functional relationships with $\bar{Y}$. By comparing to inviscid computations by the Fenton method it is evident that the streaming velocities in the boundary layers below the wave surface and above the fluid bottom contribute to a strongly enhanced forward drift velocity as well an enhanced positive Lagrangian excess period right below the wave surface and above the bottom. The effect of the nonlinearity is pronounced near the surface. Conversely, both $U_{L}$ and $T_{L}-T_{0}$ become pronounced negative in the middle of the water column. The particle paths are closed at the two vertical positions where $U_{L}=0$, in contrast to many theoretical models, e.g., Constantin (2006). The shear of the experimental drift velocity compares well to the nonlinear Fenton method and the second-order theory for $-0.9<\bar{Y} / h<-0.2$ while close to the surface the measured shear becomes more than twice that obtained by the Fenton method which again is approximately twice that of the second-order theory. There is no mass flux of the periodic experimental waves, no pressure gradient, and the average elevation within the wave train is unaltered by the wave motion. An experimental error of $U_{L}$ of $8 \%$ may be estimated by the relative magnitude of the vertical drift velocity, $V_{L}$, compared to the horizontal drift velocity, where $V_{L}$ should be zero.

The present PTV-measurements highlight the additional shear due to the streaming in the boundary layers at the bottom and below the wave surface, as well as the effect of the nonlinearity, where the shears at $\bar{Y}=0$ and $\bar{Y}=-h$ are strong and of opposite sign. They both contribute to enhancing the net forward Lagrangian drift velocity at $\bar{Y}=0,-h$ 
as well as the negative drift velocity in the water column is increased. The measured Lagrangian drift velocity, systematically in excess of the inviscid theories, indicates that the wave induced Stokes drift is significantly higher than what is commonly represented in the wave-current interaction models, see e.g. Sullivan and McWilliams (2010, eq. 4), where the primary additional effect due to the boundary layer streaming should be incorporated.

Acknowledgement. The funding by the Research Council of Norway through NFR191204/V30 and NFR231491 (the Developments in Optical Measurement Technologies DOMT) is gratefully acknowledged.

\section{References}

Andrews, D. G. and McIntyre, M. E. (1978) An exact theory of waves on a Lagrangian mean flow. J. Fluid Mech. 89, 609-646.

Ardhuin, F., Marié, L., Rascle, N., Forget, P. and Roland, A. (2009). Observation and estimation of Lagrangian, Stokes and Eulerian currents induced by wind and waves at the sea surface. J. Phys. Oceanogr. 39(11), 2820-2838.

Bagnold, R. A. (1947) Sand movement my waves: some small-scale experiments with sand of very low density. J. Inst. Civil Engng. 27(4), 447-469.

Constantin, A. (2006) The trajectories of particles in Stokes waves. Invent. math. 166, 523-535. Constantin, A. (2015) The flow beneath a periodic travelling surface water wave. J. Phys. A: Math. Theor. 48143001 (25pp).

Dalziel, S. B. (1992) Decay of rotating turbulence: some particle tracking experiments. Appl. Sci. Res. 49, 217-244.

Fenton, J.D. (1988) The numerical solution of steady water wave problems. Comput. Geosci. 14(3), 357-368.

Gjøsund, S.H. (2003) A Lagrangian model for irregular waves and wave kinematics. J. Offsh. Mech. Arctic Engng. 125, 94-102.

Groeneweg, J. and Klopman, G. (1998) Changes of the mean velocity profiles in the combined wave-current motion described in a GLM formulation. J. Fluid Mech. 370, 271-296.

Grue, J., Kolaas, J. and Jensen, A. (2014) Velocity fields in breaking-limited waves on finite depth. Eur. J. Mech. B/Fluids. 47, 97-107.

Hsu, H.-C., Chen, Y.-Y. and Wang, C-F. (2010) Perturbation analysis of short-crested waves in Lagrangian coordinates. Nonlinear analysis: Real world applications, 11, 1522-1536.

Longuet-Higgins, M. S. (1953) Mass transport in water waves. Phil. Trans. R. Soc. Lond. A 245, 535-581.

Longuet-Higgins, M. S. (1960) Mass transport in the boundary layer at a free oscillating surface. J. Fluid Mech. 8, 293-306.

Longuet-Higgins, M. S. and Stewart, R.W. (1962) Radiation stress and mass transport in gravity waves, with applications to 'surf beats'. J. Fluid Mech. 13, 481-504.

McIntyre, M.E. (1981) On the 'wave momentum myth'. J. Fluld Mech. 106, 331-347.

Monismith, S. G., Cowen, E. A., Nepf, H. M., Magnaudet, J. and Thais, L. (2007) Laboratory observations of mean flows under surface gravity waves. J. Fluid Mech. 573, 131-147.

Nepf, H.M, Cowen, E.A., Kimmel, S.J. and Monismith, S.G. (1995) Longitudinal vortices under breaking waves. J. Geophys. Res. (Oceans) 100, 16111-16221.

Stokes, G. G. (1847) On the theory of oscillatory waves. Trans. Camb. Phil. Soc. 8, 441-455.

Sullivan, P. P. and McWilliams, J. C. (2010) Dynamics of winds and currents coupled to surface waves. Annu. Rev. Fluid Mech. 42, 19-42.

Weber, J.E. and Saetra, Ø. (1995) Effect of film elasticity on the drift velocity of capillary-gravity waves. Phys. Fluids 7, 307. 
a)

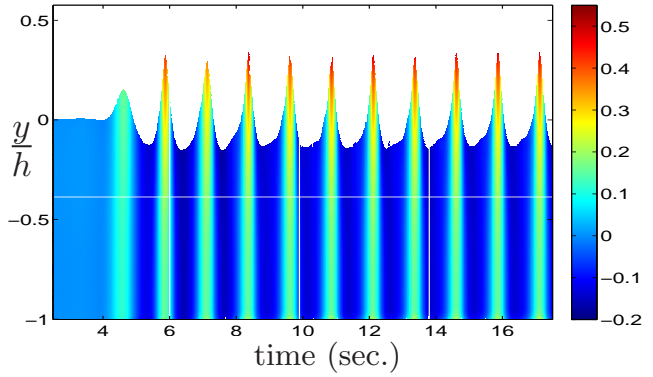

b)

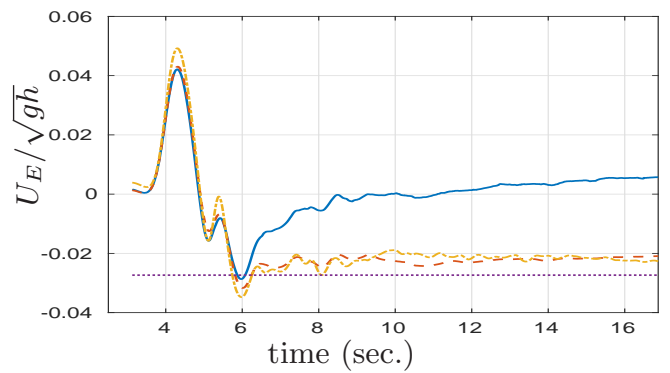

FiguRE 1. a) Elevation $y$ and maximum horizontal orbital velocity $u / \sqrt{g h}$ (color scale) vs. time in waves of height $H_{\max }=0.49 \mathrm{~h}$. White solid lines indicate recording sequences of the PTV in four intervals for $t<17.7$ sec., at two vertical levels. From Grue et al. (2014). b) Mean Eulerian horizontal velocity $\left(U_{E}\right)$ at $y / h=-0.994$ (blue solid), -0.96 (red dashed), -0.15 (yellow dash dot) and Fenton (lilac dotted).

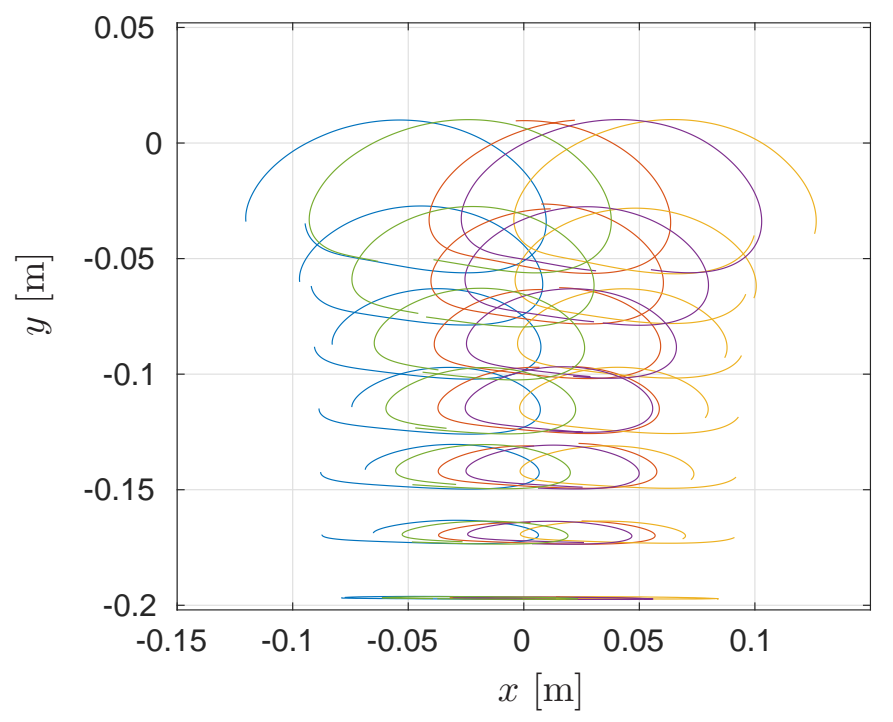

a)

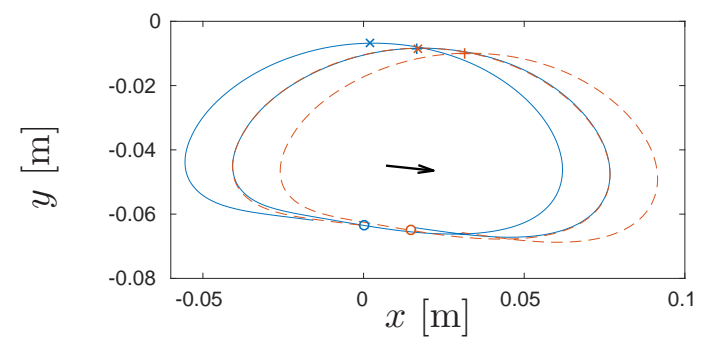

b)

FiguRe 2. a) Particle paths for several vertical positions and phases. b) Particle path for one period backward and forward integration (blue solid line) and shifted by the drift during one period (red dashed line), where black arrow indicates the drift distance $\left(x_{0}, y_{0}\right)$. Time window 1. Positions of the integration: initial (o), at $-T_{L} / 2(\times)$, at $T_{L} / 2(+)$. 

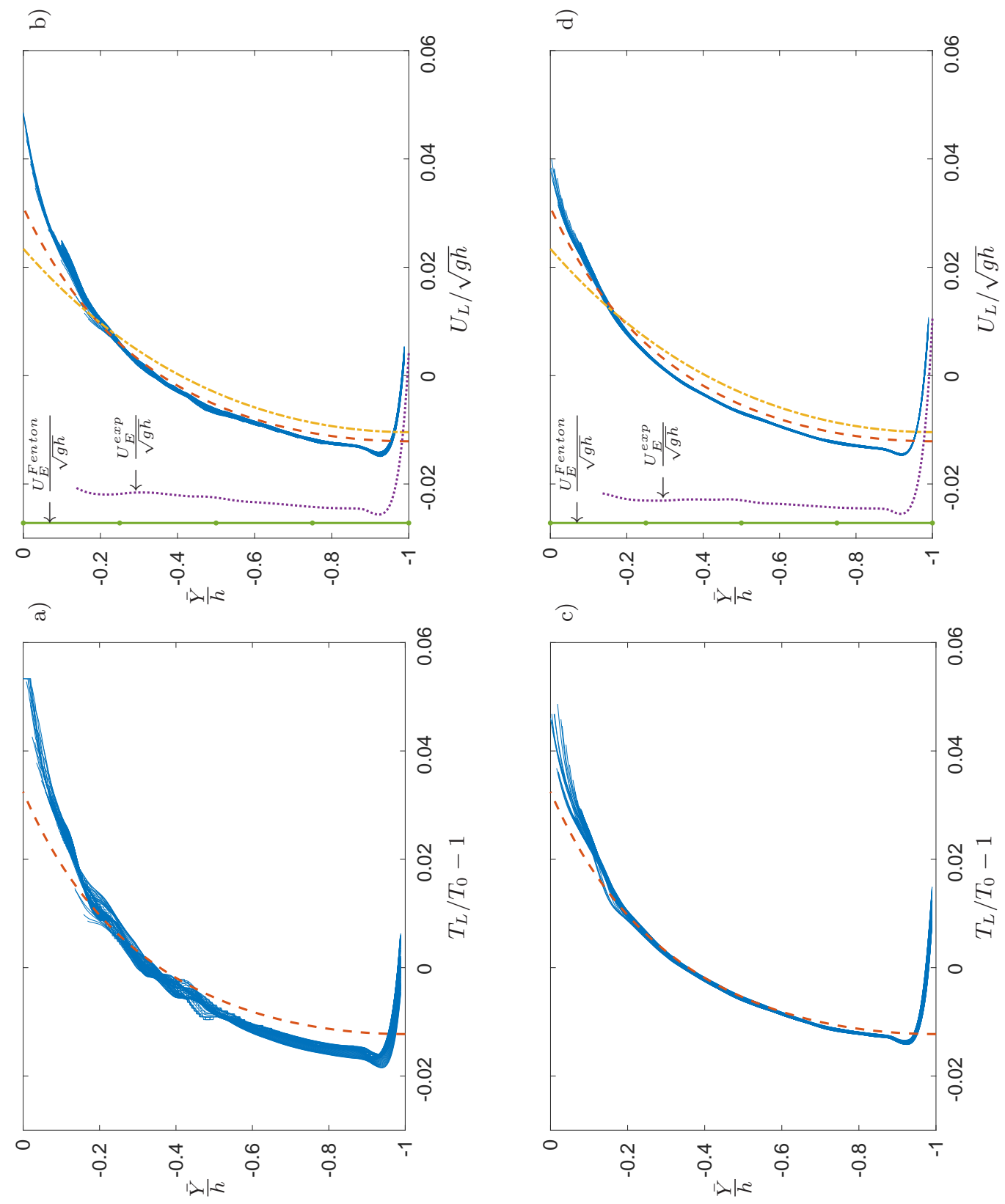

Figure 3. a) Lagrangian excess period $T_{L}-T_{0}$ and b) horizontal drift velocity $U_{L}$ for time window 1 ( $t \sim 9.9-13.8$ seconds $)$. c) $T_{L}-T_{0}$ and d) $U_{L}$ for time window $2(t \sim 13.8-17.7$ seconds). Experiments (blue symbols/line), Fenton method (dashed red), second order theory (3.4) (dashed dotted yellow). Mean Eulerian velocity $\left(U_{E}\right)$ indicated in plots b) and d). 

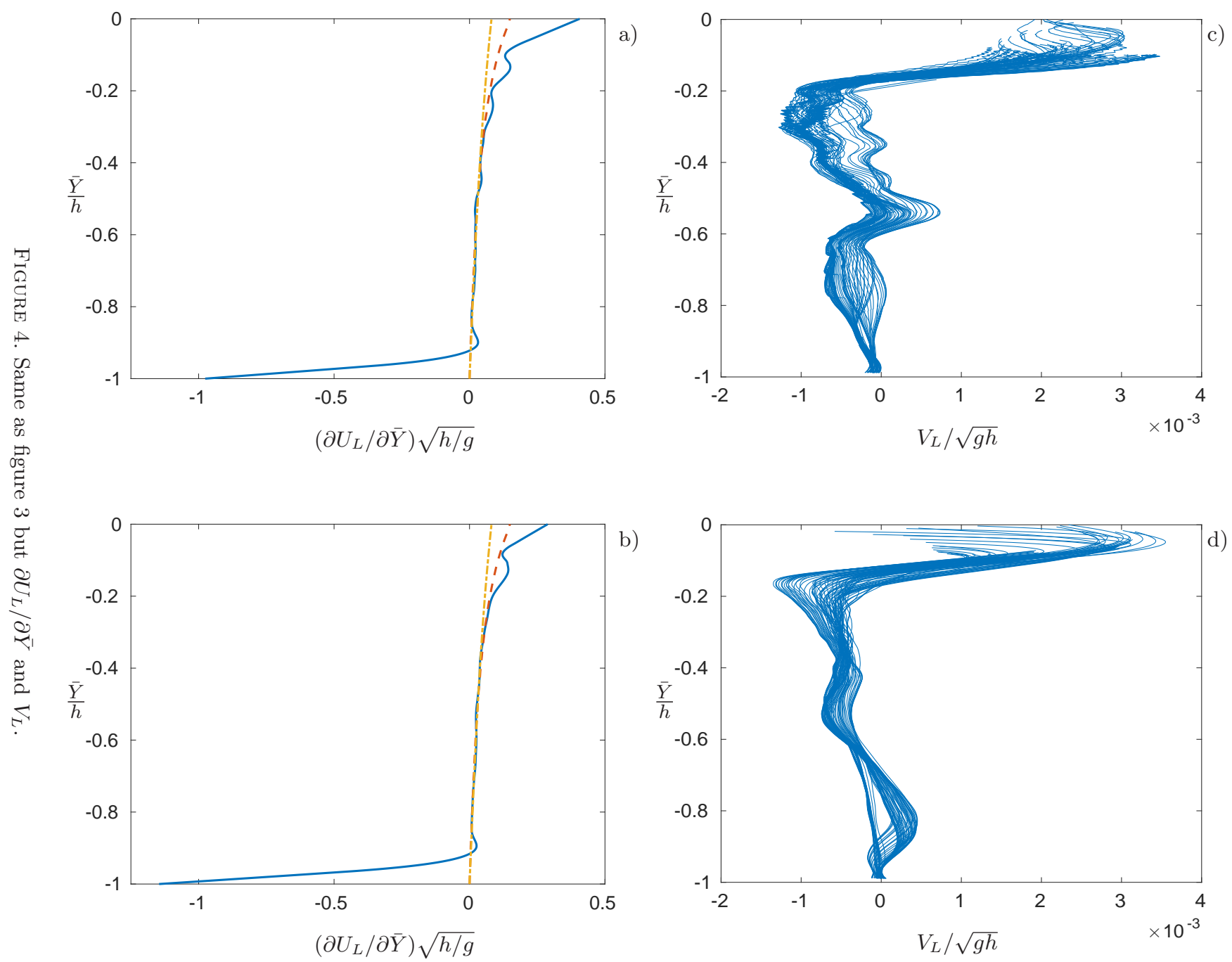

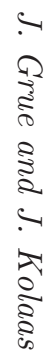

